\title{
TIME-DEPENDENT THERMO-ELASTIC CREEP ANALYSIS OF THICK-WALLED SPHERICAL PRESSURE VESSELS MADE OF FUNCTIONALLY GRADED MATERIALS
}

\author{
Mosayeb Davoudi Kashkoli, Mohammad Zamani Nejad \\ Mechanical Engineering Department, Yasouj University, Yasouj, Iran \\ e-mail:m.zamani.n@gmail.com;m_zamani@yu.ac.ir
}

\begin{abstract}
Assuming that the thermo-elastic creep response of the material is governed by Norton's law and material properties, except Poisson's ratio, are considered as a function of the radius of the spherical vessel, an analytical solution is presented for calculation of stresses and displacements of axisymmetric thick-walled spherical pressure vessels made of functionally graded materials. This analytical solution could be used to study the time and temperature dependence of stresses in spherical vessels made of functionally graded materials. Creep stresses and displacements are plotted against dimensionless radius and time for different values of the powers of the material properties.
\end{abstract}

Keywords: spherical pressure vessels, creep, time-dependent, thermo-elastic, functionally graded material

\section{Introduction}

Composites are commonly employed in various structural and engineering applications. Recently, a new class of composite materials known as functionally graded materials (FGMs) has drawn considerable attention. From viewpoints of solid mechanics, FGMs are non-homogeneous elastic mediums (Ghannad and Nejad, 2013). FGMs are those in which two or more different material ingredients change continuously and gradually along a certain direction (Noda et al., 2012). The mechanical and thermal responses of materials with spatial gradients in composition are of considerable interests in numerous industrial applications such as tribology, biomechanics, nanotechnology and high temperature technologies. These materials are usually mixtures of metal and ceramic which exhibit excellent thermal resistance with low levels of thermal stresses (Alashti et al., 2013).

Extensive studies have been carried out, both theoretically and numerically, on thermo-elastic creep stress distribution in functionally gradient materials. Time-dependent creep analysis of FGM spheres and cylinders has been an active area of research over the past decade.

Assuming the infinitesimal strain theory, Finnie and Heller (1959) studied creep problems in engineering materials and a steady-state creep solution for a spherical vessel under internal pressure. Johnson and Khan (1963) obtained a theoretical analysis of the distribution of stress and strain in metallic thick-walled spherical pressure vessels subject to internal and external pressures at elevated temperatures. Penny (1967) investigated creep of spherical shells containing discontinuities.

In this study, the solution procedure has been applied to pressurized spherical shells containing discontinuities with a view to discovering, in broad terms, how stresses change with time and how strains accumulate during the creep process. Bhatnagar and Arya (1975) obtained creep analysis of a pressurized thick-walled spherical vessel made of a homogeneous and isotropic material by making use of the finite strain theory, and with considering large strains. Miyazaki 
et al. (1977) presented a parametric analysis of the creep buckling of a shallow spherical shell subjected to uniform external pressure using the finite element incremental method. Xirouchakis and Jones (1979) investigated creep buckling behavior of a geometrically imperfect complete spherical shell subjected to a uniform external pressure using Sanders' equilibrium and kinematic equations appropriately modified to include the influence of initial stress-free imperfections in the radius.

In this study, the Norton-Bailey constitutive equations are used to describe the secondary creep behavior, and elastic effects are retained. Arya et al. (1980) studied effect of material anisotropy on creep of pressurized thick-walled spherical vessel considering the large strain theory. Kao (1981) obtained creep deformations and creep buckling times for axisymmetric shallow spherical shells with and without initial imperfections. For nonlinear creeps, both strain-hardening and time-hardening rules are employed in this study. Creep of a sphere subjected to inner and outer pressures, and also thermal stress, was discussed by Sakaki et al. (1990) by using internal stress arising from a spherically symmetric, finite plastic strain. Assuming that the elastic behavior of the material is undergoing both creep and dimensional changes, Miller (1995) presented a solution for stresses and displacements in a thick spherical shell subjected to internal and external pressure loads. Based on basic equations of steady-state creep of spherically symmetric problems, You et al. (2008) proposed a simple and efficient iterative method to determine creep deformations and stresses in thick-walled spherical vessels with varying creep properties subjected to internal pressure. Using a long-term material creep constitutive model defined by the $\Theta$ projection concept, Loghman and Shokouhi (2009) evaluated the damage histories of a thick-walled sphere subjected to an internal pressure and a thermal gradient. They studied the creep stress and damage histories of thick-walled spheres using the material constant creep and creep rupture properties defined by the theta projection concept. Aleayoub and Loghman (2010) studied time-dependent creep stress redistribution analysis of thick-walled FGM spheres subjected to internal pressure and a uniform temperature field.

In this study, using equations of equilibrium, compatibility and stress-strain relations, a differential equation, containing creep strains, for radial stress is obtained. Ignoring creep strains in this differential equation, a closed-form solution for initial thermo-elastic stresses at zero time is presented. Pankaj (2011) investigated creep stresses for a thick isotropic spherical shell by finitesimal deformation under steady-state temperature and internal pressure by using Seth's transition theory. Marcadon (2011) presented mechanical modelling of the creep behavior of Hollow-Sphere Structures. Based on basic equations of steady-state creep of spherically symmetric problems, Nejad et al. (2011) presented a new exact closed form solution for creep stresses in isotropic and homogeneous thick spherical pressure vessels. Loghman et al. (2011) investigated time-dependent creep stress redistribution analysis of thick-walled spheres made of a functionally graded material (FGM) subjected to internal pressure. In another study, Loghman et al. (2012) investigated magneto-thermo-elastic creep behavior of thick-walled spheres made of functionally graded materials (FGM) placed in uniform magnetic and distributed temperature fields and subjected to internal pressure using the method of successive elastic solution. They developed a semi-analytical method in conjunction with Mendelson's method of successive elastic solution to obtain history of stresses and strains. Assuming that the creep response of the material is governed by Norton's law, Nejad et al. (2013) presented a new exact solution for steady state creep stresses of hollow thick-walled spherical shells subjected to internal and external pressure, made of functionally graded materials (FGMs). By using the method of successive elastic solution, Fesharaki et al. (2014) presented a semi-analytical solution for the time-dependent creep behavior of hollow spheres under thermomechanical loads.

In this study, assuming that the thermo-elastic creep response of the material is governed by Norton's law, an analytical solution is presented for the calculation of stresses and displacements of FGM thick-walled spherical pressure vessels. For the creep material behavior, the solution is 
asymptotic. For the stress analysis after creeping for a long time, an iterative procedure is necessary.

\section{Geometry and loading condition, material properties and creep constitutive model}

\subsection{Geometry and loading condition}

A thick-walled spherical vessel made of a functionally graded material with inner radius $a$, and outer radius $b$, subjected to internal pressure $P_{i}$ and external pressure $P_{o}$ and a distributed temperature field due to steady-state heat conduction from the inner surface to the outer surface of the vessel is considered.

\subsection{Material properties}

The material properties are assumed to be radially dependent

$$
E(r)=E_{i}\left(\frac{r}{a}\right)^{n_{1}} \quad \alpha(r)=\alpha_{i}\left(\frac{r}{a}\right)^{n_{2}} \quad \lambda(r)=\lambda_{i}\left(\frac{r}{a}\right)^{n_{3}}
$$

Here $E_{i}, \alpha_{i}$ and $\lambda_{i}$ are the modulus of elasticity, linear expansion and thermal conductivity on the linear surface, $r=a$ and $n_{1}, n_{2}$ and $n_{3}$ are inhomogeneity constants determined empirically.

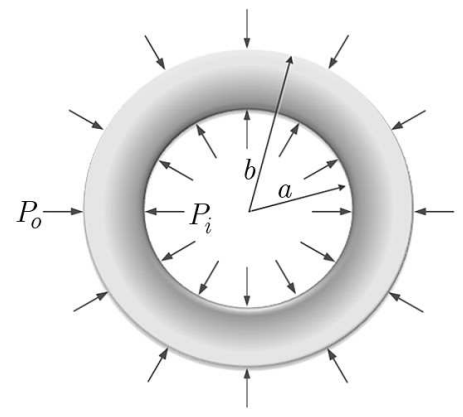

Fig. 1. Geometry and boundary conditions of the sphere

\subsection{Creep constitutive model}

For materials with creep behavior, Norton's law (1956) is used to describe the relations between the rates of stress $\dot{\sigma}_{i j}$ and strain $\dot{\varepsilon}_{i j}$ in the multi-axial form

$$
\dot{\varepsilon}_{i j}=\frac{1+\nu}{E} \dot{\sigma}_{i j}-\frac{\nu}{E} \dot{\sigma}_{k k} \delta_{i j}+\frac{3}{2} D \sigma_{e f f}^{(N-1)} S_{i j}
$$

and

$$
\begin{aligned}
& S_{i j}=\sigma_{i j}-\frac{1}{3} \sigma_{k k} \delta_{i j} \\
& \sigma_{e f f}=\sqrt{\frac{3}{2} S_{i j} S_{i j}}=\frac{1}{\sqrt{2}} \sqrt{\left(\sigma_{r r}-\sigma_{\theta \theta}\right)^{2}+\left(\sigma_{r r}-\sigma_{\phi \phi}\right)^{2}+\left(\sigma_{\phi \phi}-\sigma_{\theta \theta}\right)^{2}}=\sigma_{r r}-\sigma_{\theta \theta}
\end{aligned}
$$

where $D$ and $N$ are material constants for creep. $\sigma_{\text {eff }}$ is the effective stress, $S_{i j}$ is the deviator stress tensor and $\sigma_{r r}$ and $\sigma_{\theta \theta}=\sigma_{\phi \phi}$ are respectively the radial and circumferential stresses. 


\section{Heat conduction formulation}

In the steady-state case, the heat conduction equation for the one-dimensional problem in spherical coordinates simplifies to

$$
\frac{1}{r^{2}} \frac{\partial}{\partial r}\left(r^{2} \lambda \frac{\partial T}{\partial r}\right)=0
$$

We can determine the temperature distribution in the spherical vessel by solving Eq. (3.1) and applying appropriate boundary conditions. Equation (3.1) may be integrated twice to obtain the general solution

$$
T(r)=A_{1} r^{-n_{3}-1}+A_{2}
$$

It is assumed that the inner surface is exposed to uniform heat flux, whereas the outer surface is exposed to airstream. To obtain the constants of integration $A_{1}$ and $A_{2}$, we introduce the following boundary conditions

$$
-\lambda T^{\prime}= \begin{cases}q_{a} & \text { for } \quad r=a \\ h_{\infty}\left(T-T_{\infty}\right) & \text { for } \quad r=b\end{cases}
$$

where $T^{\prime}=d T / d r$.

Applying these conditions to the general solution, we obtain

$$
A_{1}=\frac{a^{n_{3}+2} q_{a}}{\left(n_{3}+1\right) \lambda_{i}} \quad A_{2}=T_{\infty}+\frac{q_{a}}{h_{\infty}}\left(\frac{a}{b}\right)^{2}-\frac{a^{n_{3}+2} q_{a}}{\lambda_{i}\left(n_{3}+1\right) b^{n_{3}+1}}
$$

Substituting the constants of integration $A_{1}$ and $A_{2}$ into the general solution, we obtain the temperature distribution

$$
T(r)=T_{\infty}+\frac{q_{a}}{h_{\infty}}\left(\frac{a}{b}\right)^{2}+\frac{a^{n_{3}+2} q_{a}}{\left(n_{3}+1\right) \lambda_{i}}\left(r^{-n_{3}-1}-b^{-n_{3}-1}\right)
$$

\section{Formulation of the thermo-elastic creep analysis}

\subsection{Solution for linear elastic behavior of FGM thick spherical pressure vessels}

For the stress analysis inan FGM thick spherical pressure vessel, having material creep behavior, solutions of the stresses at a time equal to zero (i.e. the initial stress state) are needed, which correspond to the solution of materials with linear elastic behavior. In this Section, equations to calculate such linear stresses in FGM thick spherical pressure vessel analytically will be given. The elastic stress-strain relations in each material read

$$
\begin{aligned}
\sigma_{r r} & =\frac{E}{(1+\nu)(1-2 \nu)}\left[(1-\nu) \varepsilon_{r r}+2 \nu \varepsilon_{\theta \theta}-(1+\nu) \alpha T\right] \\
\sigma_{\theta \theta} & =\sigma_{\phi \phi}=\frac{E}{(1+\nu)(1-2 \nu)}\left[\varepsilon_{\theta \theta}+\nu \varepsilon_{r r}-(1+\nu) \alpha T\right]
\end{aligned}
$$

where $\sigma_{r r}$ and $\sigma_{\theta \theta}=\sigma_{\phi \phi}$ are radial and circumferential stresses, respectively. Here $E, \nu$ and $\alpha$ are Young's modulus, Poisson's ratio and thermal expansion coefficient, respectively, and $T=T(r)$ is the temperature distribution in the sphere. The strain displacement relation is written as

$$
\varepsilon_{r r}=\frac{d u_{r}}{d r} \quad \varepsilon_{\theta \theta}=\frac{u_{r}}{r}
$$


where $\varepsilon_{r r}$ and $\varepsilon_{\theta \theta}=\varepsilon_{\phi \phi}$ are radial and circumferential strains and $u_{r}$ is the displacement in the $r$-direction. The equation of the stress equilibrium inside the FGM spherical pressure vessel is

$$
\frac{d \sigma_{r r}}{d r}+\frac{2}{r}\left(\sigma_{r r}-\sigma_{\theta \theta}\right)=0
$$

Using Eqs. (4.1)-(4.3), the essential differential equation for the displacement $u_{r}$ can be obtained as

$$
\begin{aligned}
& \frac{d^{2} u_{r}}{d r^{2}}+\frac{n_{1}+2}{r} \frac{d u_{r}}{d r}+\frac{\nu^{\prime \prime} n_{1}-2}{r^{2}} u_{r}=\nu^{\prime}\left(A r^{n_{2}-1}+B r^{n_{2}-n_{3}-2}\right) \\
& \nu^{\prime}=\frac{1+\nu}{1-\nu} \quad \nu^{\prime \prime}=\frac{2 \nu}{1-\nu}
\end{aligned}
$$

where

$$
\begin{aligned}
& A=\left(n_{1}+n_{2}\right)\left[\frac{T_{\infty} \alpha_{i}}{a^{n_{2}}}+\frac{q_{a} \alpha_{i}}{h_{\infty} a^{n_{2}}}\left(\frac{a}{b}\right)^{2}\right]-\frac{a^{n_{3}-n_{2}+2} q_{a} \alpha_{i}\left(n_{1}+1\right) b^{-n_{3}-1}}{\lambda_{i}\left(n_{3}+1\right)} \\
& B=\frac{\left(n_{1}+1\right) a^{n_{3}-n_{2}+2} q_{a} \alpha_{i}}{\lambda_{i}\left(n_{3}+1\right)}-\frac{a^{n_{3}-n_{2}+2} q_{a} \alpha_{i}}{\lambda_{i}}
\end{aligned}
$$

It is obvious that the homogeneous solution for Eq. (4.4) can be obtained by assuming

$$
u_{r}=C r^{x}
$$

Substituting Eq. (4.6) into Eq. (4.4) one can obtain the following characteristic equation

$$
x^{2}+\left(n_{1}+1\right) x+\left(\nu^{\prime \prime} n_{1}-2\right)=0
$$

The roots of Eq. (4.7) are

$$
\begin{aligned}
& x_{1}=\frac{-\left(n_{1}+1\right)+\sqrt{\left(n_{1}+1\right)^{2}-4\left(\nu^{\prime \prime} n_{1}-2\right)}}{2} \\
& x_{2}=\frac{-\left(n_{1}+1\right)-\sqrt{\left(n_{1}+1\right)^{2}-4\left(\nu^{\prime \prime} n_{1}-2\right)}}{2}
\end{aligned}
$$

For selected common values of $n_{1}= \pm 0.4, \pm 0.8$ and $\nu=0.292$ in this study, the discriminant of Eqs. (4.8) is always greater than zero; therefore, $x_{1}$ and $x_{2}$ are real and distinct. The homogeneous solution to Eq. (4.4) is then as follows

$$
u_{h}=C_{1} r^{x_{1}}+C_{2} r^{x_{2}}
$$

The particular solution to differential equation (4.4) can be obtained as

$$
u_{p}=u_{1} r^{x_{1}}+u_{2} r^{x_{2}}
$$

where

$$
u_{1}=\int \frac{-r^{x_{2}} P(r)}{W\left(r^{x_{1}}, r^{x_{2}}\right)} d r \quad u_{2}=\int \frac{r^{x_{1}} P(r)}{W\left(r^{x_{1}}, r^{x_{2}}\right)} d r
$$

in which

$$
P(r)=\nu^{\prime}\left(A r^{n_{2}-1}+B r^{n_{2}-n_{3}-2}\right)
$$

is the expression on the right-hand side of Eq. (4.4), and $W$ is defined as

$$
W\left(r^{x_{1}}, r^{x_{2}}\right)=\left|\begin{array}{cc}
r^{x_{1}} & r^{x_{2}} \\
x_{1} r^{x_{1}-1} & x_{2} r^{x_{2}-1}
\end{array}\right|=\left(x_{2}-x_{1}\right) r^{x_{1}+x_{2}-1}
$$


Therefore, $u_{1}$ and $u_{2}$ can be obtained by the following integration

$$
\begin{aligned}
u_{1} & =\nu^{\prime}\left[\int \frac{-r^{x_{2}} A r^{n_{2}-1}}{\left(x_{2}-x_{1}\right) r^{x_{1}+x_{2}-1}} d r+\int \frac{-r^{x_{2}} B r^{n_{2}-n_{3}-2}}{\left(x_{2}-x_{1}\right) r^{x_{1}+x_{2}-1}} d r\right] \\
& =-\nu^{\prime}\left[\frac{A r^{n_{2}-x_{1}+1}}{\left(x_{2}-x_{1}\right)\left(n_{2}-x_{1}+1\right)}+\frac{B r^{n_{2}-n_{3}-x_{1}}}{\left(x_{2}-x_{1}\right)\left(n_{2}-n_{3}-x_{1}\right)}\right] \\
u_{2} & =\nu^{\prime}\left[\int \frac{r^{x_{1}} A r^{n_{2}-1}}{\left(x_{2}-x_{1}\right) r^{x_{1}+x_{2}-1}} d r+\int \frac{r^{x_{1}} B r^{n_{2}-n_{3}-2}}{\left(x_{2}-x_{1}\right) r^{x_{1}+x_{2}-1}} d r\right] \\
& =\nu^{\prime}\left[\frac{A r^{n_{2}-x_{2}+1}}{\left(x_{2}-x_{1}\right)\left(n_{2}-x_{2}+1\right)}+\frac{B r^{n_{2}-n_{3}-x_{2}}}{\left(x_{2}-x_{1}\right)\left(n_{2}-n_{3}-x_{2}\right)}\right]
\end{aligned}
$$

Substituting Eqs. (4.14) into Eq. (4.10), one can obtain the particular solution as

$$
u_{p}=\nu^{\prime}\left[\frac{A r^{n_{2}+1}}{\left(n_{2}-x_{2}+1\right)\left(n_{2}-x_{1}+1\right)}+\frac{B r^{n_{2}-n_{3}}}{\left(n_{2}-n_{3}-x_{2}\right)\left(n_{2}-n_{3}-x_{1}\right)}\right]
$$

The complete solution to Eq. (4.4) can be written as

$$
u_{r}(r)=C_{1} r^{x_{1}}+C_{2} r^{x 2}+\nu^{\prime}\left[\frac{A r^{n_{2}+1}}{\left(n_{2}-x_{2}+1\right)\left(n_{2}-x_{1}+1\right)}+\frac{B r^{n_{2}-n_{3}}}{\left(n_{2}-n_{3}-x_{2}\right)\left(n_{2}-n_{3}-x_{1}\right)}\right]
$$

The corresponding stresses are

$$
\begin{aligned}
\sigma_{r r} & =\frac{E_{i}\left(\frac{r}{a}\right)^{n_{1}}}{(1+\nu)(1-2 \nu)}\left\{C_{1} r^{x_{1}-1}\left[2 \nu+(1-\nu) x_{1}\right]+C_{2} r^{x_{2}-1}\left[2 \nu+(1-\nu) x_{2}\right]\right. \\
& +\frac{A \nu^{\prime}\left[\left(n_{2}+1\right)(1-\nu)+2 \nu\right] r^{n_{2}}}{\left(n_{2}-x_{2}+1\right)\left(n_{2}-x_{1}+1\right)}+\frac{B \nu^{\prime}\left[\left(n_{2}-n_{3}\right)(1-\nu)+2 \nu\right] r^{n_{2}-n_{3}-1}}{\left(n_{2}-n_{3}-x_{2}\right)\left(n_{2}-n_{3}-x_{1}\right)} \\
& -(1+\nu)\left[\left(\frac{T_{\infty} \alpha_{i}}{a^{n_{2}}}+\frac{q_{a} \alpha_{i}}{h_{\infty} a^{n_{2}}}\left(\frac{a}{b}\right)^{2}-\frac{a^{n_{3}-n_{2}+2} q_{a} \alpha_{i} b^{-n_{3}-1}}{\lambda_{i}\left(n_{3}+1\right)}\right) r^{n_{2}}\right. \\
& \left.\left.+\frac{a^{n_{3}-n_{2}+2} q_{a} \alpha_{i}}{\lambda_{i}\left(n_{3}+1\right)} r^{n_{2}-n_{3}-1}\right]\right\} \\
\sigma_{\theta \theta} & =\frac{E_{i}\left(\frac{r}{a}\right)^{n_{1}}}{(1+\nu)(1-2 \nu)}\left\{C_{1} r^{x_{1}-1}\left(1+x_{1} \nu\right)+C_{2} r^{x_{2}-1}\left(1+\nu x_{2}\right)\right. \\
& +\frac{A \nu^{\prime}\left[\left(n_{2}+1\right) \nu+1\right] r^{n_{2}}}{\left(n_{2}-x_{2}+1\right)\left(n_{2}-x_{1}+1\right)}+\frac{B \nu^{\prime}\left[\left(n_{2}-n_{3}\right) \nu+1\right] r^{n_{2}-n_{3}-1}}{\left(n_{2}-n_{3}-x_{2}\right)\left(n_{2}-n_{3}-x_{1}\right)} \\
& -(1+\nu)\left[\left(\frac{T_{\infty} \alpha_{i}}{a^{n_{2}}}+\frac{q_{a} \alpha_{i}}{h_{\infty} a^{n_{2}}}\left(\frac{a}{b}\right)^{2}-\frac{a^{n_{3}-n_{2}+2} q_{a} \alpha_{i} b^{-n_{3}-1}}{\lambda_{i}\left(n_{3}+1\right)}\right) r^{n_{2}}\right. \\
& \left.\left.+\frac{a^{n_{3}-n_{2}+2} q_{a} \alpha_{i}}{\lambda_{i}\left(n_{3}+1\right)} r^{n_{2}-n_{3}-1}\right]\right\}
\end{aligned}
$$

To determine the unknown constants $C_{1}$ and $C_{2}$ in each material, boundary conditions have to be used, which are

$$
\sigma_{r r}=\left\{\begin{array}{lll}
-P_{i} & \text { for } & r=a \\
-P_{o} & \text { for } & r=b
\end{array}\right.
$$

The unknown constants $C_{1}$ and $C_{2}$ are given in Appendix. 


\subsection{Solution for creep behavior of FGM thick spherical pressure vessel}

The relations between the rates of strain and displacement are

$$
\dot{\varepsilon}_{r r}=\frac{d \dot{u}_{r}}{d r} \quad \dot{\varepsilon}_{\theta \theta}=\frac{\dot{u}_{r}}{r}
$$

And the equilibrium equation of the stress rate is

$$
\frac{d \dot{\sigma}_{r r}}{d r}+\frac{2}{r}\left(\dot{\sigma}_{r r}-\dot{\sigma}_{\theta \theta}\right)=0
$$

The relations between the rates of stress and strain are

$$
\begin{aligned}
& \dot{\sigma}_{r r}=\frac{E}{(1+\nu)(1-2 \nu)}\left[(1-\nu) \dot{\varepsilon}_{r r}+2 \nu \dot{\varepsilon}_{\theta \theta}\right]-\frac{3}{2} \frac{E}{(1-\nu)(1-2 \nu)} D \sigma_{e f f}^{(N-1)} S_{r r}^{\prime} \\
& \dot{\sigma}_{\theta \theta}=\frac{E}{(1+\nu)(1-2 \nu)}\left[\dot{\varepsilon}_{\theta \theta}+\nu \dot{\varepsilon}_{r r}\right]-\frac{3}{2} \frac{E}{(1-\nu)(1-2 \nu)} D \sigma_{e f f}^{(N-1)} S_{\theta \theta}^{\prime}
\end{aligned}
$$

where

$$
S_{r r}^{\prime}=(1-\nu) S_{r r}+2 \nu S_{\theta \theta} \quad S_{\theta \theta}^{\prime}=S_{\theta \theta}+\nu S_{r r}
$$

Using Eqs. (4.19)-(4.22), the essential differential equation for the displacement rate $\dot{u}_{r}$ in FGM spherical vessel can be obtained as

$$
\begin{aligned}
& \frac{d^{2} \dot{u}_{r}}{d r^{2}}+\frac{d \dot{u}_{r}}{d r}\left(\frac{2}{r}+\frac{d \ln E}{d r}\right)+\frac{\dot{u}_{r}}{r}\left(\frac{2 \nu}{1-\nu} \frac{d \ln E}{d r}-\frac{2}{r}\right)=\frac{d \ln E}{d r} \frac{3}{2(1-\nu)} D \sigma_{e f f}^{(N-1)} S_{r r}^{\prime} \\
& +\frac{1}{1-\nu} \frac{d}{d r}\left(\frac{3}{2} D \sigma_{e f f}^{(N-1)} S_{r r}^{\prime}\right)+\frac{3}{r(1-\nu)} D \sigma_{e f f}^{(N-1)}\left(S_{r r}^{\prime}-S_{\theta \theta}^{\prime}\right)
\end{aligned}
$$

In general, the quantities $\sigma_{\text {eff }}, S_{r r}^{\prime}$ and $S_{\theta \theta}^{\prime}$ are very complicated functions of the coordinate $r$, even in an implicit function form. Therefore, it is almost impossible to find an exact analytical solution to Eq. (4.23). We can alternatively find an asymptotical solution to Eq. (4.23). At first, we assume that $\sigma_{e f f}, S_{r r}^{\prime}$ and $S_{\theta \theta}^{\prime}$ are constant, i.e. they are independent of the coordinate $r$

$$
\frac{d^{2} \dot{u}_{r}}{d r^{2}}+\frac{1+n_{1}}{r} \frac{d \dot{u}_{r}}{d r}-\frac{\nu n_{1}-1}{r^{2}} \dot{u}_{r}=\frac{3}{2} \frac{D}{r} \sigma_{e f f}^{(N-1)}\left[S_{r r}^{\prime}\left(1+n_{1}-v^{\prime}\right)+S_{\theta \theta}^{\prime}\left(n_{1} \nu^{\prime}-1+\nu^{\prime}\right)\right]
$$

The homogeneous solution to Eq. (4.24) is then

$$
u_{h}=D_{1} r^{x_{1}}+D_{2} r^{x_{2}}
$$

The particular solution to differential equation (4.24) can be obtained as

$$
u_{p}=u_{1}^{\prime} r^{x_{1}}+u_{2}^{\prime} r^{x_{2}}
$$

where

$$
\begin{aligned}
u_{1}^{\prime} & =\int \frac{-r^{x_{2}} H r^{-1}}{\left(x_{2}-x_{1}\right) r^{x_{1}+x_{2}-1}} d r \quad u_{2}^{\prime}=\int \frac{r^{x_{1}} H r^{-1}}{\left(x_{2}-x_{1}\right) r^{x_{1}+x_{2}-1}} d r \\
H & =\frac{3}{2} \frac{D}{(1-\nu)} \sigma_{\text {eff }}^{(N-1)}\left[S_{r r}^{\prime}\left(2+n_{1}\right)-2 S_{\theta \theta}^{\prime}\right]
\end{aligned}
$$

The complete solution to Eq. (4.24) can be written as

$$
\dot{u}_{r}(r)=D_{1} r^{x_{1}}+D_{2} r^{x_{2}}+\frac{3}{2} \frac{D r \sigma_{e f f}^{(N-1)}\left[\left(n_{1}+2\right) S_{r r}^{\prime}-2 S_{\theta \theta}^{\prime}\right]}{(1-\nu)\left[n_{1}\left(\nu^{\prime \prime}-1\right)-2\right]}
$$


where the unknown constants $D_{1}$ and $D_{2}$ can be determined from the boundary conditions. The corresponding stress rates are

$$
\begin{aligned}
\dot{\sigma}_{r r} & =\frac{E_{i}\left(\frac{r}{a}\right)^{n_{1}}}{(1-2 \nu)(1+\nu)}\left\{D_{1} r^{x_{1}-1}\left[2 \nu+(1-\nu) x_{1}\right]+D_{2} r^{x_{2}-1}\left[2 \nu+(1-\nu) x_{2}\right]\right. \\
& \left.+\frac{3}{2} \frac{\nu^{\prime}}{n_{1}\left(\nu^{\prime \prime}-1\right)-2} D \sigma_{e f f}^{(N-1)}\left[\left(n_{1}+2\right) S_{r r}^{\prime}-2 S_{\theta \theta}^{\prime}\right]-\frac{3}{2} D \sigma_{e f f}^{(N-1)} S_{r r}^{\prime}\right\} \\
\dot{\sigma}_{\theta \theta} & =\frac{E_{i}\left(\frac{r}{a}\right)^{n_{1}}}{(1-2 \nu)(1+\nu)}\left\{D_{1} r^{x_{1}-1}\left(1+\nu x_{1}\right)+D_{2} r^{x_{2}-1}\left(1+\nu x_{2}\right)\right. \\
& \left.+\frac{3}{2} \frac{1+\nu}{n_{1}\left(\nu^{\prime \prime}-1\right)-2} D \sigma_{e f f}^{(N-1)}\left[\left(n_{1}+2\right) S_{r r}^{\prime}-2 S_{\theta \theta}^{\prime}\right]-\frac{3}{2} D \sigma_{e f f}^{(N-1)} S_{\theta \theta}^{\prime}\right\}
\end{aligned}
$$

To determine the unknown constants $D_{1}$ and $D_{2}$ in each material, boundary conditions have to be used. Since the inside and outside pressures do not change with time, the boundary conditions for stress rates on the inner and outer surfaces may be written as

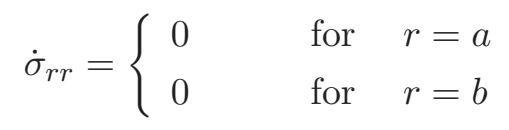

The unknown constants $D_{1}$ and $D_{2}$ are given in Appendix. When the stress rate is known, the calculation of stresses at any time $t_{i}$ should be performed iteratively

$$
\sigma_{i j}^{(i)}\left(r, t_{i}\right)=\sigma_{i j}^{(i-1)}\left(r, t_{i-1}\right)+\dot{\sigma}_{i j}^{(i)}\left(r, t_{i}\right) d t^{(i)} \quad t_{i}=\sum_{k=0}^{i} d t^{(k)}
$$

To obtain a generally useful solution, a higher-order approximation of $\sigma_{e f f}, S_{r r}^{\prime}$ and $S_{\theta \theta}^{\prime}$ should be made

$$
\begin{aligned}
& \sigma_{\text {eff }}(r)=\sigma_{\text {eff }}(\bar{r})+\frac{\left.\frac{d}{d r}\left[\sigma_{\text {eff }}(r)\right]\right|_{r=\bar{r}}}{1 !}(r-\bar{r})+\frac{\left.\frac{d^{2}}{d r^{2}}\left[\sigma_{\text {eff }}(r)\right]\right|_{r=\bar{r}}}{2 !}(r-\bar{r})^{2}+\frac{\left.\frac{d^{3}}{d r^{3}}\left[\sigma_{\text {eff }}(r)\right]\right|_{r=\bar{r}}}{3 !}(r-\bar{r})^{3} \\
& S_{r r}^{\prime}(r)=S_{r r}^{\prime}(\bar{r})+\frac{\left.\frac{d}{d r}\left[S_{r r}^{\prime}(r)\right]\right|_{r=\bar{r}}}{1 !}(r-\bar{r})+\frac{\left.\frac{d^{2}}{d r^{2}}\left[S_{r r}^{\prime}(r)\right]\right|_{r=\bar{r}}}{2 !}(r-\bar{r})^{2}+\frac{\left.\frac{d^{3}}{d r^{3}}\left[S_{r r}^{\prime}(r)\right]\right|_{r=\bar{r}}}{3 !}(r-\bar{r})^{3} \\
& S_{\theta \theta}^{\prime}(r)=S_{\theta \theta}^{\prime}(\bar{r})+\frac{\left.\frac{d}{d r}\left[S_{\theta \theta}^{\prime}(r)\right]\right|_{r=\bar{r}}}{1 !}(r-\bar{r})+\frac{\left.\frac{d^{2}}{d r^{2}}\left[S_{\theta \theta}^{\prime}(r)\right]\right|_{r=\bar{r}}}{2 !}(r-\bar{r})^{2}+\frac{\left.\frac{d^{3}}{d r^{3}}\left[S_{\theta \theta}^{\prime}(r)\right]\right|_{r=\bar{r}}(r-\bar{r})^{3}}{3 !}
\end{aligned}
$$

where $\bar{r}$ is the center point of the wall thickness in the following analysis.

\section{Numerical results and discussion}

In the previous Sections, the analytical solution of creep stresses for FGM thick-walled spherical vessels subjected to uniform pressures on the inner and outer surfaces has been obtained. In this Section, some profiles are plotted for the radial displacement, radial stress and circumferential stress as a function of the radial direction and time. An FGM thick-walled spherical vessel with creep behavior under internal and external pressure is considered. Radii of the sphere are $a=20 \mathrm{~mm}, b=40 \mathrm{~mm}$. Mechanical properties of the sphere such as modulus of elasticity, linear expansion and thermal conductivity are assumed to be varying through the radius. The inhomogeneity constants $n_{1}=n_{2}=n_{3}=n$, and $n$ ranges from -0.8 to +0.8 . The following data for loading and material properties are used in this investigation: $E_{i}=207 \mathrm{GPa}$, 
$\nu=0.292, \alpha_{i}=10.8 \cdot 10^{-6} \mathrm{~K}^{-1}, P_{i}=80 \mathrm{MPa}, P_{o}=0 \mathrm{MPa}, q_{a}=500 \mathrm{~W} / \mathrm{m}^{2}, \lambda_{i}=43 \mathrm{~W} /\left(\mathrm{m}^{\circ} \mathrm{C}\right)$, $h_{\infty}=6.5 \mathrm{~W} /\left(\mathrm{m}^{2}{ }^{\circ} \mathrm{C}\right), T_{\infty}=25^{\circ} \mathrm{C}, D=1.4 \cdot 10^{-8}, N=2.25$.

The distributions of creep stress components $\sigma_{r r}$ and $\sigma_{\theta \theta}$ after $10 \mathrm{~h}$ of creeping for values of $n= \pm 0.4, \pm 0.8$ are plotted in Fig. 2. It must be noted from Fig. 2a that the radial stress increases as $n$ decreases, and that the radial stress for different values of $n$ is compressive. The absolute maximums of radial stress occur at the outer edge. It means the maximum shear stress, which is $\tau_{\max }=\left(\sigma_{\theta \theta}-\sigma_{r r}\right) / 2$ for each value of $n$ will be very high on the outer surface of the vessel.

(a)

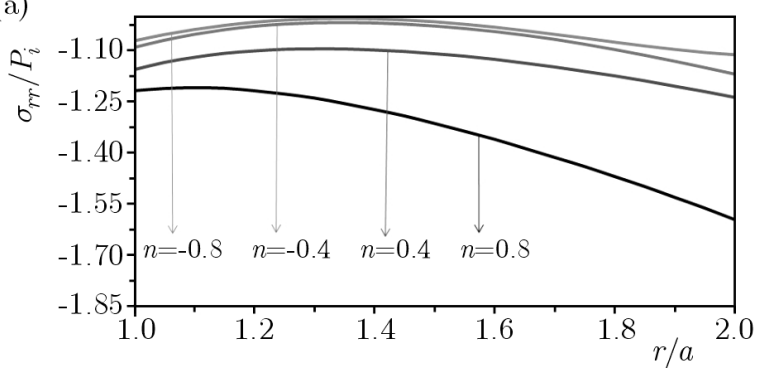

(b)

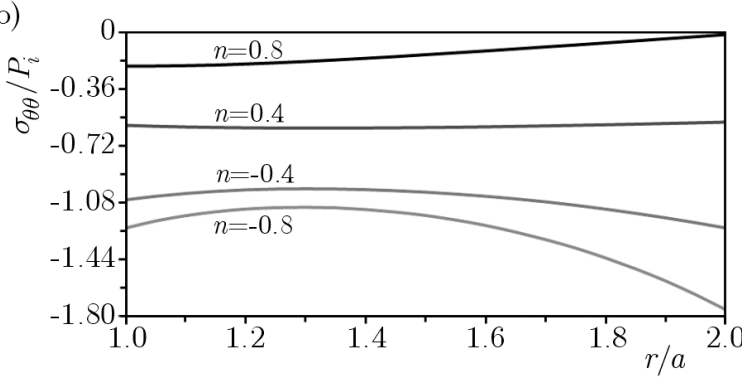

Fig. 2. Normalized radial and circumferential stresses versus dimensionless radius after $10 \mathrm{~h}$ of creeping

It is also clear from Fig. 2a that the maximum changes in the radial stresses with time take place for the material $n=0.8$ and the minimum changes occur for $n= \pm 0.4,-0.8$. The circumferential stress shown in Fig. $2 \mathrm{~b}$ remains compressive throughout and is observed to decrease with the increasing radius for $n=-0.4,-0.8$, and reaches the minimum value somewhere towards the inner radius followed by an increase with a further increase in the radius. It also can be seen from Fig. $2 \mathrm{~b}$ that the circumferential stress remains compressive throughout the cylinder for $n=+0.8$ with the maximum value at the inner radius and zero at the outer radius under the imposed boundary conditions, and that the minimum changes occur for $n=0.4$.

Time dependent stress redistributions at the point $r=30 \mathrm{~mm}$ are shown in Fig. 3. It can be seen in Fig. 3a that the radial stress increases as time increases. It must be noted from Fig. 3b that, for $n=+0.4,+0.8$ the circumferential stress decreases as time increases, whereas for $n=-0.4,-0.8$ the circumferential stress increases as time increases.
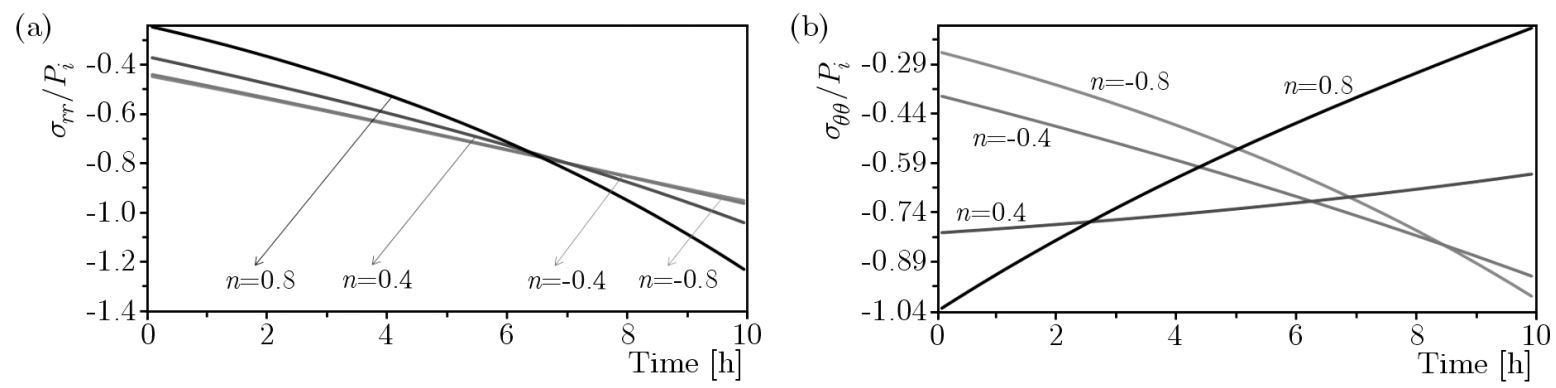

Fig. 3. Time-dependent radial and circumferential stresses at the point $r=30 \mathrm{~mm}$

The radial displacement along the radius is plotted in Fig. 4a. There is an increase in the value of the radial displacement as $n$ increases and the maximum value of radial displacement occurs at the outer edge. The time-dependent radial displacement at the point $r=30 \mathrm{~mm}$ is shown in Fig. 4b. Figure 4b shows that the radial displacement redistribution at the point 
$r=30 \mathrm{~mm}$ increases as time increases for $n=-0.4,-0.8$, while for $n=+0.4,+0.8$ the radial displacement decreases as time increases. Figure 5 shows the effect of adding external pressure to the radial and circumferential stresses. It can be seen in Fig. 5 that the radial stress decreases as the external pressure increases while the circumferential stress increases as the external pressure increases.
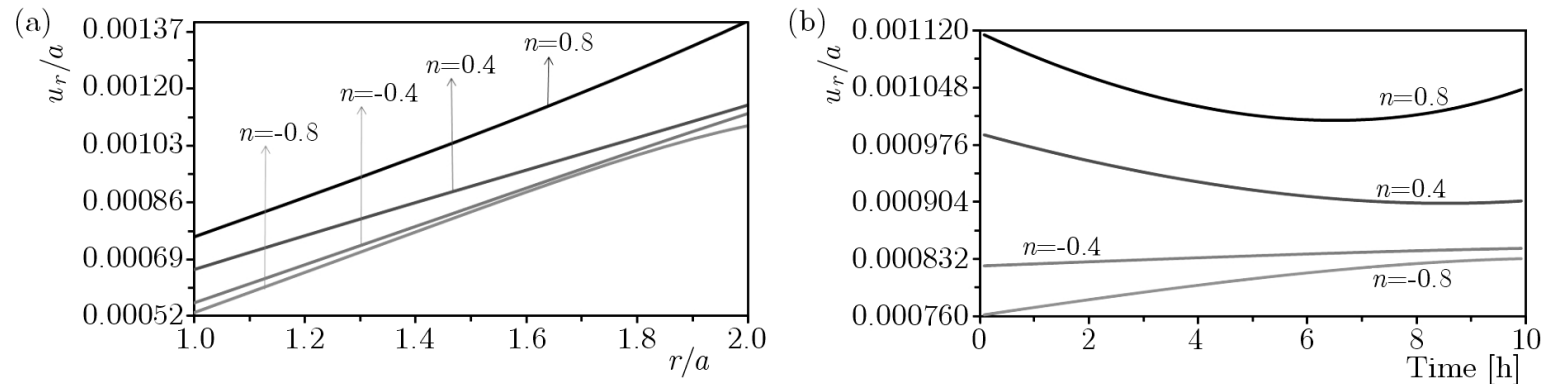

Fig. 4. (a) Normalized radial displacement versus dimensionless radius after $10 \mathrm{~h}$ of creeping, (b) time-dependent radial displacement at the point $r=30 \mathrm{~mm}$

(a)

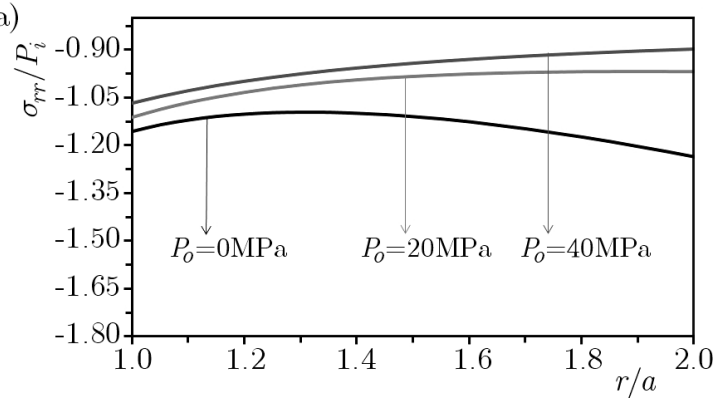

(b)

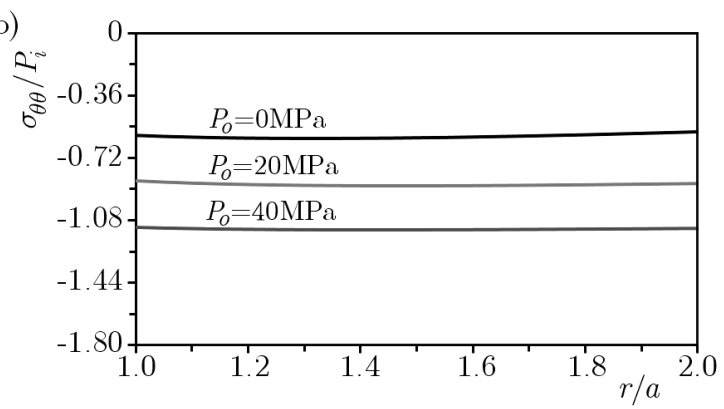

Fig. 5. The effect of adding external pressure to the radial and circumferential stresses

Temperature distribution of four different values of $n$ is shown in Fig. 6. It can be seen in Fig. 6 that the maximum values of temperature occur at the inner radius for $n=-0.8$ and that the minimum values of temperature occur at the outer radius for all values of $n$ under the imposed boundary conditions.

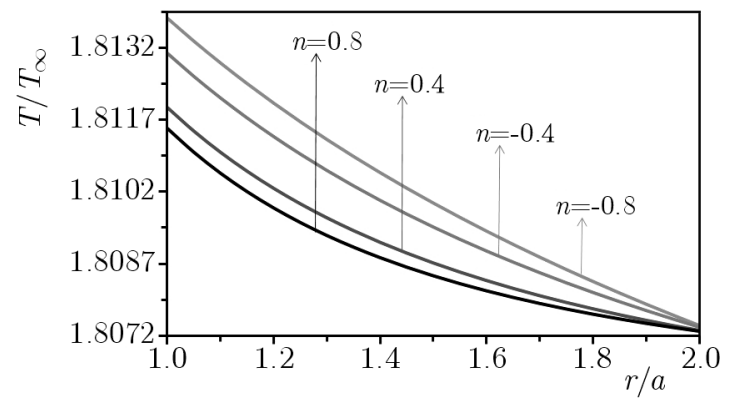

Fig. 6. Temperature distribution of FGM thick-walled spherical vessel for values of $n= \pm 0.4, \pm 0.8$

\section{Conclusions}

In this paper, assuming that the thermo-creep response of the material is governed by Norton's law, an analytical solution is presented for the calculation of stresses and displacements of FGM thick-walled spherical pressure vessels. For the stress analysis inasphere, having material creep 
behavior, the solutions of the stresses at a time equal to zero (i.e. the initial stress state) are needed, which corresponds to the solution of materials with linear elastic behavior. It is assumed that the material properties change as graded in the radial direction to a power law function. To show the effect of inhomogeneity on the stress distributions, different values are considered for inhomogeneity constants. The pressure, inner radius and outer radius are considered constant. The heat conduction equation for the one-dimensional problem in spherical coordinates is used to obtain temperature distribution in the sphere. For the creep material behavior, the solution is asymptotic. For the stress analysis after creeping for a long time, the iterative procedure is necessary. It could be seen that the inhomogeneity constants have significant influence on the distributions of the creep stresses and radial displacement. By increasing the grading parameter $n$, the normalized radial stress increases due to internal pressure and temperature distribution while the normalized circumferential stress decreases (Fig. 2). The absolute maximums of radial and circumferential stresses occur at the outer edge. It must be noted that the radial and circumferential stresses at the point $r=30 \mathrm{~mm}$ for different values of $n$ are compressive. As can be seen, the absolute maximum of radial stress at the point $r=30 \mathrm{~mm}$ occurs at a time equal to 10 hours for different values of $n$, whereas for $n=+0.4,+0.8$ the absolute maximum of circumferential stress occurs at a time equal to zero, and for $n=-0.4,-0.8$ the absolute maximum of circumferential stress occurs at a time equal to 10 hours.

\section{Appendix}

The unknown constants in Eqs. (4.17) are

$$
\begin{aligned}
C_{1} & =\frac{-P_{i}(1+\nu)(1-2 \nu)}{E_{i}\left[2 \nu+(1-\nu) x_{1}\right] a^{x_{1}-1}}-\frac{C_{2}\left[2 \nu+(1-\nu) x_{2}\right] a^{x_{2}-1}}{\left[2 \nu+(1-\nu) x_{1}\right] a^{x_{1}-1}} \\
& -\frac{A \nu^{\prime}\left[\left(n_{2}+1\right)(1-\nu)+2 \nu\right] a^{n_{2}}}{\left(n_{2}-x_{2}+1\right)\left(n_{2}-x_{1}+1\right)\left[2 \nu+(1-\nu) x_{1}\right] a^{x_{1}-1}} \\
& -\frac{B \nu^{\prime}\left[\left(n_{2}-n_{3}\right)(1-\nu)+2 \nu\right] a^{n_{2}-n_{3}-1}}{\left(n_{2}-n_{3}-x_{2}\right)\left(n_{2}-n_{3}-x_{1}\right)\left[2 \nu+(1-\nu) x_{1}\right] a^{x_{1}-1}} \\
& +\frac{1+\nu}{\left[2 \nu+(1-\nu) x_{1}\right] a^{x_{1}-1}}\left\{\left[\frac{T_{\infty} \alpha_{i}}{a^{n_{2}}}+\frac{q_{a} \alpha_{i}}{h_{\infty} a^{n_{2}}}\left(\frac{a}{b}\right)^{2}-\frac{a^{n_{3}-n_{2}+2} q_{a} \alpha_{i} b^{-n_{3}-1}}{\lambda_{i}\left(n_{3}+1\right)}\right] a^{n_{2}}\right. \\
& \left.+\frac{a q_{a} \alpha_{i}}{\lambda_{i}\left(n_{3}+1\right)}\right\} \\
C_{2}= & \frac{\left(P_{i} b^{x_{1}-1}-\frac{P_{o} a^{n_{1}+x_{1}-1}}{b^{n_{1}}}\right)(1+\nu)(1-2 \nu)}{E_{i}\left[2 \nu+(1-\nu) x_{2}\right]\left(b^{x_{2}-1} a^{x_{1}-1}-b^{x_{1}-1} a^{x_{2}-1}\right)} \\
& -\frac{A \nu^{\prime}\left[\left(n_{2}+1\right)(1-\nu)+2 \nu\right]\left(n_{2}+\nu^{\prime}+1\right)\left(b^{n_{2}} a^{x_{1}-1}-b^{x_{1}-1} a^{n_{2}}\right)}{\left(n_{2}-x_{2}+1\right)\left(n_{2}-x_{1}+1\right)\left[2 \nu+(1-\nu) x_{2}\right]\left(b^{x_{2}-1} a^{x_{1}-1}-b^{x_{1}-1} a^{x_{2}-1}\right)} \\
& -\frac{B \nu^{\prime}\left[\left(n_{2}-n_{3}\right)(1-\nu)+2 \nu\right]\left(b^{n_{2}-n_{3}-1} a^{x_{1}-1}-b^{x_{1}-1} a^{n_{2}-n_{3}-1}\right)}{\left(n_{2}-n_{3}-x_{2}\right)\left(n_{2}-n_{3}-x_{1}\right)\left[2 \nu+(1-\nu) x_{2}\right]\left(b^{x_{2}-1} a^{x_{1}-1}-b^{x_{1}-1} a^{x_{2}-1}\right)} \\
& +\frac{1+\nu}{\left[2 \nu+(1-\nu) x_{2}\right]\left(b^{x_{2}-1} a^{x_{1}-1}-b^{x_{1}-1} a^{x_{2}-1}\right)}\left\{\left[\frac{T_{\infty} \alpha_{i}}{a^{n_{2}}}+\frac{q_{a} \alpha_{i}}{h_{\infty} a^{n_{2}}}\left(\frac{a}{b}\right)^{2}\right.\right. \\
& \left.-\frac{a^{n_{3}-n_{2}+2} q_{a} \alpha_{i} b^{-n_{3}-1}}{\lambda_{i}\left(n_{3}+1\right)}\right]\left(b^{n_{2}} a^{x_{1}-1}-b^{x_{1}-1} a^{n_{2}}\right) \\
& \left.+\frac{q_{a} \alpha_{i}}{\lambda_{i}\left(n_{3}+1\right)}\left(b^{n_{2}-n_{3}+1} a^{n_{3}-n_{2}+x_{1}+1}-b^{x_{1}-1} a\right)\right\}
\end{aligned}
$$


The unknown constants in Eqs. (4.29) are

$$
\begin{aligned}
D_{1} & =\frac{\frac{3}{2} \nu^{\prime} D \sigma_{e f f}^{(N-1)}\left(a^{x_{2}-1}-b^{x_{2}-1}\right)\left[\left(n_{1}+2\right) S_{r r}^{\prime}-2 S_{\theta \theta}^{\prime}\right]}{\left[2 \nu+(1-\nu) x_{1}\right]\left[n_{1}\left(\nu^{\prime \prime}-1\right)-2\right]\left(a^{x_{1}-1} b^{x_{2}-1}-b^{x_{1}-1} a^{x_{2}-1}\right)} \\
& +\frac{\frac{3}{2} D \sigma_{e f f}^{(N-1)} S_{r r}^{\prime}\left(b^{x_{2}-1}-a^{x_{2}-1}\right)}{\left[2 \nu+(1-\nu) x_{1}\right]\left(a^{x_{1}-1} b^{x_{2}-1}-b^{x_{1}-1} a^{x_{2}-1}\right)} \\
D_{2} & =\frac{\frac{3}{2} \nu^{\prime} D \sigma_{e f f}^{(N-1)}\left(a^{x_{1}-1}-b^{x_{1}-1}\right)\left[\left(n_{1}+2\right) S_{r r}^{\prime}-2 S_{\theta \theta}^{\prime}\right]}{\left[2 \nu+(1-\nu) x_{2}\right]\left[n_{1}\left(\nu^{\prime \prime}-1\right)-2\right]\left(a^{x_{2}-1} b^{x_{1}-1}-b^{x_{2}-1} a^{x_{1}-1}\right)} \\
& +\frac{\frac{3}{2} D \sigma_{e f f}^{(N-1)} S_{r r}^{\prime}\left(b^{x_{1}-1}-a^{x_{1}-1}\right)}{\left[2 \nu+(1-\nu) x_{2}\right]\left(a^{x_{2}-1} b^{x_{1}-1}-b^{x_{2}-1} a^{x_{1}-1}\right)}
\end{aligned}
$$

\section{References}

1. Alashti R.A., Khorsand M., Tarahhomi M.H., 2013, Three-dimensional asymmetric thermoelastic analysis of a functionally graded rotating cylindrical shell, Journal of Theoretical and Applied Mechanics, 51, 1, 143-158

2. Aleayoub S.M.A., Loghman A., 2010, Creep stress redistribution analysis of thick-walled FGM spheres, Journal of Solid Mechanics, 2, 10, 115-128

3. Arya V.K., Debnath K.K, Bhatnagar N.S., 1980, The spherical vessel with anisotropic creep properties considering large strains, International Journal of Non-Linear Mechanics, 15, 185-193

4. Bhatnagar N.S., Arya V.K., 1975, Creep of thick-walled spherical vessels under internal pressure considering large strains, Indian Journal of Pure and Applied Mathematics, 6, 10, 1080-1089

5. Bhatnagar N.S., Pradnya K., Arya V.K., 1987, Primary creep analysis of an anisotropic thick-walled spherical shell, Journal of Pressure Vessel Technology, 109, 347-351

6. Finnie I., Heller W.R., 1959, Creep of Engineering Materials, McGraw-Hill Book Co. Inc., New York

7. Ghannad M., Nejad M.Z., 2013, Elastic solution of pressurized clamped-clamped thick cylindrical shells made of functionally graded materials, Journal of Theoretical and Applied Mechanics, $\mathbf{5 1}, 4,1067-1079$

8. Fesharaki J., Loghman A., Yazdipoor M., Golabi S., 2014, Semi-analytical solution of timedependent thermomechanical creep behavior of FGM hollow spheres, Mechanics of Time-Dependent Materials, 18, 41-53

9. Johnson A.E., Khan B., 1963, Creep of metallic thick-walled spherical vessels subject to pressure and radial thermal gradient at elevated temperatures, International Journal of Mechanical Sciences, $\mathbf{5}, 507-532$

10. KAO R., 1981, Nonlinear creep buckling analysis of initially imperfect shallow spherical shells, Computers and Structures, 14, 1/2, 111-122

11. Loghman A., Aleayoub S.M.A., Hasani Sadi M., 2012, Time-dependent magnetothermoelastic creep modeling of FGM spheres using method of successive elastic solution, Applied Mathematical Modelling, 36, 836-845

12. Loghman A., Ghorbanpourarani A., Aleayoub S.M.A., 2011, Time-dependent creep stress redistribution analysis of thick-walled functionally graded spheres, Mechanics of Time-Dependent Materials, 15, 353-365

13. Loghman A., Sнокоuнi N., 2009, Creep damage evaluation of thick-walled spheres using a long-term creep constitutive model, Journal of Mechanical Science and Technology, 23, 2577-2582 
14. Marcadon V., 2011, Mechanical modelling of the creep behaviour of hollow-sphere structures, Computational Materials Science, 50, 3005-3015

15. Miller G.K., 1995, Stresses in a spherical pressure vessel undergoing creep and dimensional changes, International Journal of Solids and Structures, 32, 14, 2077-2093

16. Miyazaki N., Yagawa G., Ando Y., 1977, A parametric analysis of creep buckling of a shallow spherical shell by the finite element method, Nuclear Engineering and Design, 41, 257-263

17. Nejad M.Z., Hoseini Z., Niknejad A., Ghannad M., 2011, A new analytical solution for creep stresses in thick-walled spherical pressure vessels, Journal of Basic and Applied Scientific Research, 1, 11, 2162-2166

18. Nejad M.Z., Hoseini Z., Taghizadeh T., Niknejad A., 2013, Closed-form analytical solution for creep stresses of pressurized functionally graded material thick spherical shells, Advanced Science Letters, 19, 2, 464-467

19. Noda N., Ootao Y., Tanigawa Y., 2012, Transient thermoelastic analysis for a functionally graded circular disk with piecewise power law, Journal of Theoretical and Applied Mechanics, 50, $3,831-839$

20. PAnkAJ T., 2011, Creep transition stresses of a thick isotropic spherical shell by finitesimal deformation under steady-state of temperature and internal pressure, Thermal Science, 15, 2, 157-165

21. Penny R.K., 1967, The creep of spherical shells containing discontinuities, International Journal of Mechanical Sciences, 5, 373-388

22. Sakaki T., Kuroki T., Sugimoto K., 1990, Creep of a hollow sphere, Journal of Applied Mechanics, Transactions ASME, 57, 276-281

23. Xirouchakis P.C., Jones N., 1979, Axisymmetric and bifurcation creep buckling of externally pressurized spherical shells, International Journal of Solids and Structures, 16, 131-148

24. You L.H., Ou H., Zheng Z.Y., 2008, Steady-state creep analysis of thick-walled spherical pressure vessels with varying creep properties, Journal of Pressure Vessel Technology, 130, 0145011$-0145015$ 